\title{
HERPETOFAUNA PERUANA: UNA VISION PANORAMICA SOBRE INVESTIGACION, CONSERVACION Y MANEJO ${ }^{1}$.
}

ANTONIO W. SATAS

\author{
Deparamemo de Herpetwiopha. \\ Mueo de Hiuturis Natamil \\ Uaiveridad Nosoral Mayoe de Soa Marcos.
} Av. Aetnakes 1256, Apibo. 140434, Lime 14, FERUP.

\author{
RESUMEN
}

Se revisa el avance de las iavestigacioes en herpetofauna peruana de las datimes 25 años. El incremento de la diversidad de especies de anfibios y reptiles de los ültimos afos creció acejeradameane, pero no çveió en igual proporción el conocimiento en cuasto a su biologia. ecrología Y especialmente, su biogoografla. Se dan a conoetr las actuales medidas de manejo y problemas de conservación y se propooen una serie de reconsendaciones para el estbilecimiento de un adecuade programa de investigación y manejo para su conservación.

PALARRAS CLAVE: Peri, Herpetofauna, buodiversidal conservacion, manejo of fauna

\section{SUMMARY}

The research advances of the last 25 years in peruvian herpetofauna is revised. At difference with the knowledgment on biology and ecology of its species, the iscrease in species richness growths accelerated. The current practices of conservation and management are presented, and some recomendations to establish an actualized research and conservation program are offered.

KEY WORDS: Peru, Herpetofauna, biodiversity, conservation, wildlife managernent.

El Perí, por la amplia gama de ecosistemas con que cuenta, es considerado como uno de los países poseedores de mayor diversidad de flora y fauna a nivel mundial (McNeely y col, 1990). Esto es realmente cierto en el caso de los anfibios y reptiles. El Instituto de Recursos Mundiales, listaba hasta 1989 para Perú, 235 especies de anfibios y 297 de reptiles, ubicándolo en quinto lugar entre los países con mayor diversidad herpetofaunal en el Mundo, después de paises como Brasil, Colombia, Ecuador y México (World Resources Institute 1990). Muy recientemente estos valores se ban incrementado, concciéndose que en el caso de los anfibios hasta hace poco, el número era de 315 (Rodriguez y col. 1993) para actualmente ser más de 340 (DAPTF-Peru, datos no publicados); estimándose que sucede de igual manera con el número de especies de reptiles. Sin embargo, se espera que estos valores aumenten aún más, pues se debe mencionar que los otros países indicados cuentan a su fayor con una abrumadora diferencia en cuanto al número de investigaciones realizadas, y no es hasta muy reciente que nuestro pais ha sido visitado con mayor frecuencia por investigadores extranjeros.

\section{INVESTIGACION}

El conocimiento de la amplia diversidad herpetofaunal del Perú, ha concitado el interés cientifico de investigadores de todo el mundo durante las dos últimas décadas, cuyos trabajos en muy diferentes campos, se han visto expresados en un amplio número de publicaciones.

Varios géneros han sido objeto de revisión sistemática de sus especies, principalmente por investigadores extranjeros, entre los más importantes podemos cirar para los anfibios los de los géneros andinos Gastrotheca (Duellman y Fritts, 1972), Pleuradema (Duellman y Veloso, 1977). Phrynopus (Lynch, 1975) y Telmatobius (Wiens, 1993; Sinsch y col., 1995); asi como los géneros amazónicos Hemiphractus (Trueb, 1974), Leprodactylus (Heyer, 1994) y el ampliamente distribuido Physalaemus (Cannatella y Duellman, 1984); también deben mencionarse las revisiones de los géneros y especies de la familia Centrolenidae (Duellman, 1976), las de especies del género Rana grupo palmipes (Hillis y De Sá, 1988) y las de la familia Dendrobatidae (Zimmennann y Zimmermann, 1988).

Entre los saurios algunos génetos han recibido igual atención, pudiendo citarse entre los mís importantes las revisiones de los géneros costeros Phyllodactylus (Dixon y Huey, 1970) y Tropiduras (Dixon y Wright, 1975), los géneros andincsStenocercus (Fritts, 1974) y Pholidobolus (Montanucci, 1973); asi como los amazónicos Bachia e Iphisa (Dixon, 1973; 1974). Entre los ofidios pueden citarse las revisiones de Boideos (Carrillo, 1968) y de las serpientes de las familias Viperidac, Elapidae e Hidrophiidae (Carrillo, I985). 
Numerosas nuevas especies han sido descritas para estos y otros géneros, especialmeate en los últimos años; por ejemplo, para formas de anfibios de ambientes premontanos, pueden citarse los géneros Atelopus (Gray y Cannatella, 1985), Bufo (Duellman y Ochoa, 1991, Duellman y Schulte, 1993) y Centrotenella (Duellman. 1976; Cannatella y Duellman, 1982; Cadle y McDiarmid, 1990); en ambientes amazónicos pueden citarse los géneros Epipedobates (Rodriguez y Myers, 1993). Dendrobates (Myers, 1979: Ainchinger, 1991; Morales, 1992). Hyla (Henle, 1991; Duellman y Trueb, 1989; Duellman 1982), Phyllomediesa (Duellman et al. 1988), Scarthyla (Duellman y De Sá, 1988), Scinax (Duellman y Wiens, 1992; Duellman y Wiens, 1993), Jschnocnema (Duellman.1990b) y Phyllontastes (Duellman, 1991b); Gastrotheca (Dueliman, 1987b; Duellman y Trueb. 1988; Duellman y Fritts, 1972), Phrynopus (Cannatella, 1984; Hedges, 1990) y Telmatobius (Morales, 1988) para las regiones andinas. Especial mención debe hacerse de los géneros Eleutherodactylus (Ducllman, 1978a, 1978 b. 1990a, 1991a, 1992a, 1992b; Duellman y Wild. 1993. Lynch y Lescure, 1980, Hedges y Schluter, 1992 Rodriguez, 1994), para el cual se han descrito numerosas nuevas especies para diversas regiones del pafs, asi cotno para el género Rana (Hillis y De Sá, 1988) que nos permite citar dos de sus especies para Perú, como verdaderos representantes de la familia Ranidae.

Entre los reptiles, las descripciones de tres especies de saurios y dos de ofidios pueden citarse como las adiciones más recientes: las de especies de lagartijas de los géneros Alopoglossas (Duellman, 1973), Enyalioides (Dueliman, 1973) Macropholidus (Cadle y Chuna, 1995), y las de los colúbridos Sibynomorphnes williamsii (Carrillo, 1974) y Coniophanes longiques (Cadle, 1989)

Otro enfoque de investigación importante es el de los listados o inventarios locales y regionales de biodiversidad. Entre los más importantes que se pueden citar tenemos para nuestra Amazonía norte los realizados para lquitos y sus alrededores por Dixon y Soini $(1975,1977)$, para las localidades de Colonia y Yuvineto por Lescure y Gase (1986), asi como las de Orellana y Contamana por Hocking y Salas (datos no publicados); para la amazonía central los realizados en la Estación Biológica Panguana (Schluter, 1983; Schluter y Salas, 1990) y los de la Cordillera del Sira (Duellman y Toft, 1979).

Para la amazonía sur pueden meecionarse los realizados en la Estación Biológica Cocha Cashu (Rodriguez y Cadle. 1991: Salas 1991a) y la Estacióa Biológica Pakitza (McDiarmid, datos atin no publicados), ambas en el Parque Nacional Manu; la Zona Reservada Tambopata Candamo (Mc Diarmid, dates no publicados: Conservation International. 1994) y el Santuario Nacional Pampas del Heath (Morales, 1991; Icochea, 1992); y por último, la Reserva de Cuzco Amazónico (Ducilman y Salas, 1991).
En los andes y regiones premontanas puede citarse los trabajos de Rodriguez en el Parque Nacional Rio Abiseo (Datos aún no publicados); los de la Cordillera de Huancabamba (Duellman y Wild, 1993); Salas para la región de Ancash (Datos aún no publicados); Hocking y Hocking para el Parque Nacional Yanachaga Chemillen (Hocking, comunicación personal); Sinsch (1986, 1990) para la sierra central; Péfaur y colaboradores (1978a. 1978b) para Arequipa. También deben mencionarse los de Reynolds y Salas a lo largo de un transecto elevacional entre Puucartambo y Atalayu, en Cuzco y Madre de Dios (datos aún no publicados).

Para la extensa región costera al oeste de los andes. puede citarse a Cuadros para Matapalo, una localidad casi limitrofe con Ecuador (Cuadros, com pers..): Salas y colaboradores en Tumbes y Piura (Salas, 1991b) y Cadle (1991) para Bosgue Monte Seco en el nor-occidente peruano.

Estos ban permitido ahadir varios nuevos registros de especies para nuestro país (Carrillo 1985, 1987; Duellman y Salas 1991) y ampliar nuestro conocimiento sobre sus distribuciones. Es de esperar que su número se incremente aún más en los próximos an̂os con la publicación de resultados de investigaciones y descrip. ciones de nuevas especies actualmente en ejecución (Salas y Sinsch, en prensa). Sin embargo, es aún incompleto el conocimiento sobre la diversidad total existente en varias de estas localidades y regiones, debido a que varias de ellas corresponden a programas de investigación de corta duración, lo que no garantiza el registro de la totalidad de especies presentes. Por ello es de comprender que es aún más pobre nuestro conocimiento sobre la diversidad de nuestro país, debido principalmente a su amplio, diverso y accidentado territorio, (ver fig. 1)

Numerosos trabajos de investigación han sido realizados en el campo de la historia natural, como por ejemplo sobre biologia y conducta reproductiva en anuros ( Salas, 1990b; Schliter, 1987; Schluter, 1990; Schluter y Salas, 1990; Sinsch, 1985), asi como de reptiles OMittermeier y col, 1978: Ascencios 1990; Fachín, 1992; Soini, 1994); termoregulación en anfibios y reptiles (Sinsch, 1989; Sinsch, 1991: Salas, en prep.). conducta de orientaciós (Sinsch, 1988) y bioacústica también de anuros (Schluter 1979, 1980; Sinsch y Joerman, 1989; Heyer y Morales 1995), dieta larval (Guevara y Morales, 1991), asi como de relaciones de comensalismo entre anuros e invertebrados (Cocroft y Hambler, 1989).

Aspecto importante representa la ecología de comunidades de anuros y saurios (Aichinger, 1987, 1991; Huey, 1974, 1979, Péfaur y López-Tejeda. 1983; Duellman, 1987; Péfaur y Duellman, 1977; Salas, 1991 a. Toft y Duellman, 1979, Toft 1980, Rodriguez, 1994), la variación intrapoblacional y geogrifica en coloración en 


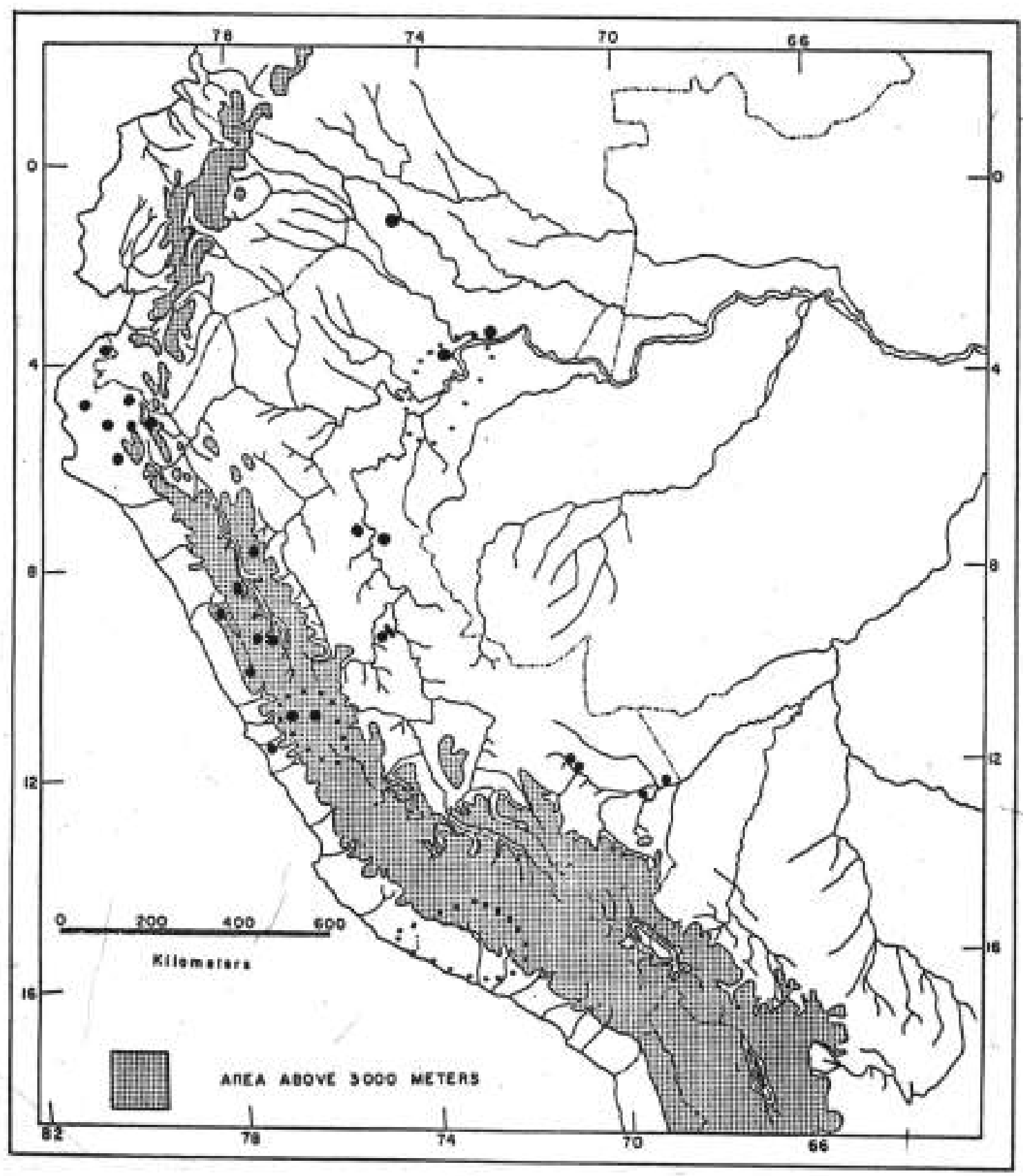

Fig 1. Mapa del Peni mostrando las principales localidades y dretas que han sido objeto de inventarios de diversidad de arfibios y reptiles en los ilfimas 25 avios. 
especies de los géneros Edalorhina e Hyla (Duellman y Morale5, 1990. Titus et al, 1989), la biogeografía de especies selectas (Morales, 1988) y el estado de conservación de especies de quelonios acuáticos y ofidios (Fachín ,1991; Schulte, 1988; Aranda y Chandler, 1989; Soini et al. 1989 y Vásquez, 1983).

En morfologia y fisiología pueden citarse los trabajos sobre desarrollo osteológico de anuros (De Sá y Trueb. 1991), morfología de las expansiones digitales de anuros (Hertwig y Sinsch, 1995), los de convergencia adaptativa en lagartijas geckonide (Carritlo et al. 1990) y las descripciones larvales de anfibios (Cadle y Altig. 1991).

Por último debe mencionarse un listado de nombres populares de reptiles peruanos (Carrillo, 1990). Sin embargo, numerosos trabajos permanecen igualmente como tesis o informes de proyectos no publicados, pérdiendose la información por la falta de difusión (Herron, 1985).

Sin embargo, a la luz de nuestros avances sobre el conocimiento de la amplia diversidad de especies y comunidades presentes, se hace evidente que este es aún incompleto en taxonomia, sistemática, zoogeografía, biología y ecologia, pudiendo decirse que existe aún mucho por conocer de nuestra herpetofauna.

\section{APROVECHAMIENTOYCONSERVACIONDE ESPECIES}

Varias especies se utilizan desde tiempo indeterminado de manera artesanal por pobladores locales, de muy diversas maneras. Por ejemplo, entre los anfibios, se emplean para alimentación por los pobladores andinos la Rana de Junín (Batrachophrynus macrostomus) y las ranas Telmatobius culews y $T$. arequipensis; los sapos Bufo marinus y , Leptodactylus pentadacrylus son utilizados de la misma manera entre comunidades nativas de la umazonfa. Entre los reptiles tenemos los saurios costeros como el Cañán (Dicnodon guttulatum y D. holmbergi), el Pacazo o Capazo (Iguana iguina) y la Iguana negra (Callopistes flavipunctatus) (Salas, 1991b). No es necesario abundar más en detalles de similar uso de las diferentes especies de quelonios y lagartos de nuestra amazonia, que tambien son capturados en los últimos años por el alto valor de su piel. Información importante sobre la situación de estos grupos de especies han sido publicadas por diversos autores (Aranday Chandler, 1989; Soini et al. 1989 y Vásquez, 1983).

En general, el consumo de estas especies se debe a que se les atribuye propiedades curativas para diversos males. Asi, en medicina folklórica (curanderismo), es común el uso de los Telmatobinidos en toda la sierra, pues, se les atribuye a la curación de enfermedades de las vias respiratorias y del sistema nervioso.

Tabla 1. Clasificación penaana de especies amenazadas y en peligro de la fauna silvestre. (Set.1990).

\section{A $\mathbf{R}$}

c Bolitoglossa peruviand m

Crocodylus acutus

b) Especies en situación vulnerable.

Batrachophrynus brachydactylus

Telmatobius rima

Podocnemis expansam

Podocnemis umifilis

Dermochelys coriacea

Chelonia mydas

Eretmochelys imbricata

Caretta caretta

Lepidachelys olivacea

Boa constrictor

Eunectes murinus

Caiman crocodilus

Melanosuchas niger

c) Especies en situación rara.

Paleosuchus palpebrosus

Padacnemis sextuberculata

d) Especies en situación indeterminada.
Bufo dapsilis

Ceratophrys stolemanic

Eleutherodactylus cajamarcensis

Elestherodactylus bymani

$m$ Gastrotheca ochoai

$m$ Leptadactyius elenae

c Physalaemus pustulatus

c Syncope antenori

c. Telmatobius culeus

Telmatobius marmoratus

Trachycephalus jordani

$c, m$ Chelus fimbriatus

m Phrynops geoffroanus

m Phrynops nasutus

m Phrynops rufipes

Platemys platycephala

Kinostermum leucostomum

m Kinosternum scorpioides

m Geochelone carbonaria

Epicrates cenchria

Paleosuchus trigonatus
A

R

1)

c

a

a

a

m

c

In

a.

a

c

III

III

III)

m

In

In

III

II

In

m

A: anfibios, R: Repriles, c: costero, a: andino, mi amozónico. 


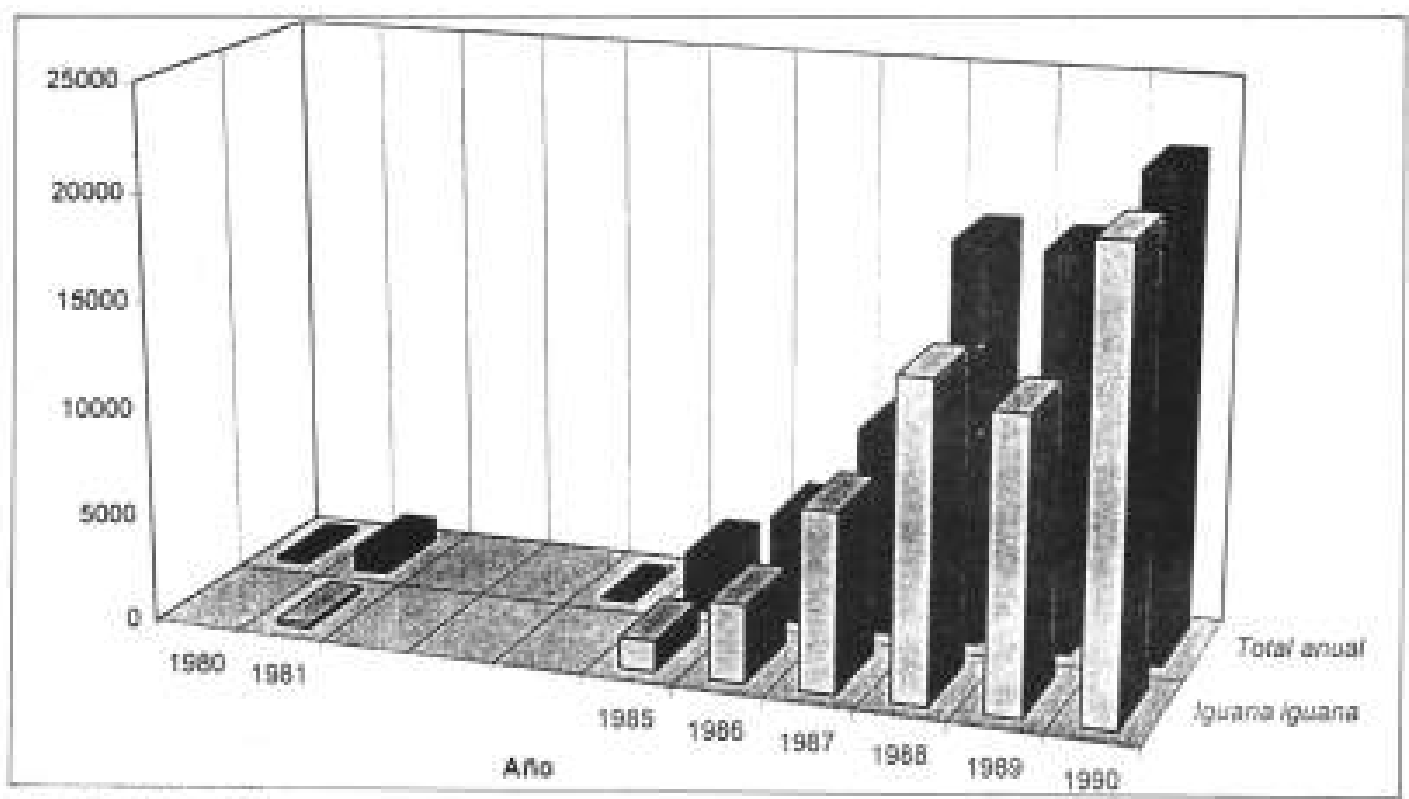

Fig. 2 Cuadro de las exporfaciones rotales de herpetofauna y de lipuana iguana de arios selectas entre 1980 y 1990 . Los datos para 1990 camprenden sólo las estadisticas para las 7 primeror meses del ahlo.

En los últimos siete años, ha aparecido un auevo modo de aprovechamiento de las especies de nuestra herpetofauna: la exportación comercial como mascotas. Prícticamente desarrollada en muy pequeña escala hasta 1985, se ha incrementado significativamente afectando a un total de 28 especies (Tabla 1), sin que se conozca el minimo de información necesaria sobre las poblaciones $y$ su impacto.

Para dar una idea de sus alcances, en la fig. 2 se muestra el número total de cjemplares de anfibios y reptiles exportados durante el año 1980 y el periodo 1985-1990 (Salas, 1991b). Tal como puede observarse, la exportación comercial de anfibios y reptiles se mantuvo con ligera variación entre 1980 y 1985, para incrementarse aceleradamente, llegando en tan sólo la primera mitad de 1990, a un total de más de 22,000 ejemplares, de los cuales, más del 95 \% fueron de Pacazo (Iguana iguana), cuyos ejemplares fueron exportados a valores que oscilaban entre U.S.\$1.50 y $\$ 10.00$ por ejemplar. Aún más, debe mencionarse que entre las exportaciones figuran siete especies de la selva amazónica, especies que por ley están prohibidas indefinidamente para fises comerciales (D.S. N 934-73AG), siendo una de ellas Dendrobates hystrionicus, cuya distribución está citada para Colombia y Ecuador y que no presenta poblaciones registradas para Perd (Frost, 1985).

Más del $55 \%$ de estos ejemplares va con destino a los Estados Unidos, pero debe tambiér mencionarse como otros importantes países receptores a Alemania, Japon y Canada, en los que son muy cotizados debido a las facilidades de espacio que requieren para su mantenimiento, llegando por ejemplo un ejemplar juvenil de Iguana iguana a ser ofrecido por U.S.\$\$0.00 y un adulto por entre U.S.\$100 y $\$ 150,00$, una Bod constrictor por $\$ 200.00$ y una Ameiva ameiva por $\$ 30,00$ (Observaciones personales).

Extas diversas formas de aprovechamiento han originado una presión sobre las poblaciones de las especies mencionadas, cuyo efecto no puede ser estimado en-su totalidad por la carencia de trabajos a largo plazo sobre estas especies, a excepción de pocos casos conocidos. Uno de estos. es el de la Rana de Junín, especie que ba sido sobre-explotada pura consumo local y regional, por el atractivo que representa como aljmento exótico. Las comparaciones de datos de captura actual con los registrados por el Proyecto Explotación y Fomento de la Rana (Varios autores, 1970), dan a notar que existe una disminación del tamaťo promedio de los ejemplares capturados, como un indicativo de la poca recuperación que se le brinda a las poblaciones de la especie en el lago; a lo anterior puede sumarse el efecto de contaminantes y el manejo artificial del nivel de agua, agravado últimamente, por las sequias ocurridas en los últimos años (Salas, datos no publicados).

A lo anterior, se une ahora un nuevo problema, especialmente en el caso de los anfibios: la evidencia registrada de declinación poblacional de varias de sus especies en todo el mundo (Blaustein y Wake 1990a, 1990b; Phillips, 1990; Wake, 1990). Se han llevado a cabo varias reuniones de especialistas para tratar sobre el tema (Jutterbock, 1990, Anónimo, 1991); por la información reunida es evidente que este constituía un fenómeno global, no delimitado a una región geográfica ni a todos los taxa presentes en cuda localidad, asi como no existir un único factor causal planetario conocido. Como resultado se propusieron varias recomendaciones: 
1. Son necesarios realizar estudios a largo plazo de poblaciones de anfibios, con énfasis en conocer el rol en los ecosistemas y biología de población, así como sus relaciones con los factores físicos y químicos del ambiente.

II. Recopilación de información y uso de datos históricos.

III. Diseño y desarrollo de programas educacionales dirigidos al público par dar a conocer el estado e importancin de las especies de anfibios (Jutterbock. 1990).

Evidencias de tales declinas han sido presentadas con reportes para Venezuela (La Marca citado por Duelliman, en Jutterbock 1990), Ecuador (Coloma, comunicación personal) y Pert (Duellman en Jutterbock 1990); citándose observaciones en especies de los géneros Atelopus, Eleathenadacfylus, Telmatobius y Colostethus, génetos con especies en nuestro pais. Actualmente, la Unión Mundial por la Conservacion (IUCN) ha creado un equipo de trabajo especial bajo la dirección de la Comision para la Supervivencia de Especies (SSC), el Declining Amphibian Populations Task Force (DAPTF), con coordinadores en diversos paises de todo el mundo y que cuenta inclusive con un Newsletter, el FROGLOG (IUCN/SSC Task Force on Declining Amphibians 1992).

Dos investigadores peruanos fueron designados como Coordinadores por Perú para esta comisión internacional, habiendo organizado hasta la fecha diez grupos de trabajo local en las principales ciudades del país, asi como dos talleres, uno en Lima y otro en Piura, contribuyendo de esta manera a una mayor difusión del problema y a una mejor coordinación de los esfuerzos para su estudio. Como resultado de estos talleres, se ha elaborado una lista de 346 especies de anfibios para el pars, asi como la designación de seis de ellas como en situación de declinación pohlacional (Vial y Saylor, 1993).

Esto contrasta con las acciones gubernamentales de conservación. La lista del Gobiemo Peruano de especies de fauna amenazadas y en peligro (Tabla 2), incluye 170 especies de fauna silvestre, de las cuales 14 son de anfibios y 24 de reptiles (CDC, 1990). Entre las incluidas se encuentran especies que han sido objeto de protección por entidades de conservación a nivel internacional, pero igualmente otras que por 50 abundancia y amplia distribución no debieron ser incluidas. como por ejemplo los casos de Batrachophnnus brachydactyius. Telmatobius rimac. Eleutherodactylus lymani $6 E$. cajamarcensis. Es poco el esfuerzo hecbo con objeto de considerar, en la elaboración de la lista existente, información actualizada del real estado poblacional de las especies presentes en nuestro territorio, en base a la consulta con los especialistas o investigadores de campo, verdaderos conocedores del tema.

Es así como se deja de incluir especies como el Gecko de Lima (Phyllodactylus sentosius), una lagartija cuyo estado de conservación debe ser considerado en vías de extinción, ya que su distribución abarcaba lo que un día fue el valle del Rímac, actualmente rodeado $y$ ocupado por lo que es hoy la ciudad de Lima; $\delta$ la Boa Esmeralda (Corallus caninus), verdadera expresión de belleza entre los reptiles, cazada injustamente por su parecido a la temida "Loro machaco" (Bothriopsis bilineata), vibora arbórea venenosa: 6 la Rana de Junín Batrachophrymus macrostomus, especie amenazada por razones ya expuestas anteriormente.

Tabla 2. Lista de Especies de Anfibios y Reptiles sujetos a exportación entre 1985-1991 (Extraída de Salas 1990b) , costero=c, andino=a, amazónicos $=m$.

\author{
ANFIBIOS: \\ Bufo marinus c.m \\ Bufo spinulosies a \\ Eleutherodactylas \\ cajamancensis , a \\ Dendrobates spp. , c,m \\ REPTILES: \\ (Saurios) \\ Ameiva edracantha, $\mathrm{c}$ \\ Callopisfes favipunctatas ic \\ Dicrodon gurtulatum, $\mathrm{c}$ \\ Dicrodion heterolepis , c \\ Enyaloides palpebrosus, in \\ Iguana iguana , c.m \\ Liolaenas mulfiformis, a \\ Ophnoessoides iridescens, c
}

Phyllodacrylas reisst, $c$

Polychnus femoralis, c

Stenocercus omatissimas,c

Tropidures accipitalis, $\mathrm{C}$

Tropidurus peravianies , $\mathrm{c}$

Tropidunus tigris, $\mathrm{c}$

\section{Ondios}

Boa constrictor, c,m

Bothrops spp.,c,m

Drymarchon corais, c, m

Epicnates cenchria, m

Leptophis ahaetalla, m

Micrurus mertensi, c

Oxybelis fiulgidus, $c$

Oxyrhopus firzingeri, c

Pseustes sp., m

Alsophis elegans, c 


\section{MANEJO DE HERPETOFAUNA}

Son prácticamente nulas las iniciativas de manejo de especies que se hallan llevado a cabo en nuestro pais. Entre los programas exitosos para otros países, pueden citarse el de la Rana Toro (Rana catesbeiana). desarrollado en Brasil, que contaba ya hasta 1990, con 100,000 hectáreas de terreno dedicadas a instalaciones de ranigranjas; su producción para entonces, cubría únjeamente la demanda nacional y contaba con muy buenas perspectivas para el mercado internacional. gracias al interés por las ancas de rana, por países principalmente europeos. En el caso de los reptiles. pueden citarse los programas de manejo de la Iguana verde ( . iguana) en Costa Rica (conocido en Perú como Pacazo), programa que ha llevado a cabo ya reintroducciones exitosas de ejemplares hacia el medio natural (Gruson, 1989; Rojas, 1991); el de la Iguana Colorada Tupinambis rufescens iniciado reciéntemente en Argentina ó el del Cocodrilo del Nilo (Crocodylus niloticus), llevado a cabo exitosamente en Zimbawe. Africa (Hutton y Child 1989).

Los pocos intentos realizados en Perú, comprenden el proyecto de Explotación y Fomento de la Rana de Junín Batrachophrynus macrostomus, emprendido en dos oportunidades por el Ministerio de Pesquería en colaboración con investigadores de la Univ. Nac. Mayor de San Marcos (Anónimo, 1976; Salas, 1986) y el programa de manejo con fines de conservacióa de tortugas acuáticas. llevado a cabo en diversas especies de nuestra Amazonía (Soini et al. 1989). Sin embargo las posibilidades de aprovechamiento para nuestra berpetofauna presente son muy amplias, abarcando campos como alimentación, medicina o mascotas y su mancjo puede garantizar un aprovechamiento sostenido de sus especies, dentro de los lineamientos modernos de la conservación.

\section{RECOMENDACIONES}

La información expaesta da a notar un escaso conocimiento de nuestra herpetofauna y la necesidad de implementar una adecuada estrategia de investigación. manejo y conservación de sus especies. Para su implementación se sugieren cinco directivas básicas:

\footnotetext{
1. Implementar Inventarios de Especies de Anfibios y Reptiles para Perú.

Nuestro conocimiento actual está basado principalmente en los trabajos de Frost (1986) y Duellman (1979) para los anfibios; Peters y Donoso-Bartos (1970) para los saurios, Peters y Orejas-Miranda (1970) para los ofidios, Carrillo (1970) y Vanzolini (1986), trabajos que comprenden listados a nivel mundial o regional de especies de anfibios y reptiles. En tal sentido, actualmente
}

miembros del Departamento de Herpetología del Museo de Historia Natural de la Universidad Nacional Mayor de San Marcos, están elaborando listas de la herpetofauna existente (Rodríguez y col, 1993: Carrillo, com. pers.); y en el mismo sentido viene desarrollándose un programa de inventario computarizado en el Centro de Datos de Conservación. La elaboración de estos listados, con el aporte de otros realizados a nivel local (Miranda, Martin. Hurtado ef al., comunicaciones personales) permitirán contar con un conocimiento básico actualizado, de la diversidad y distribución de especies presentes.

\section{Evaluación de la Información existente sobre el estado de las poblaciones $y$ de sus especies.}

Deberá comprender la evaluación de toda la información existente, que incluya tanto las publicaciones de descripción de especies, como los trabajos de tesis o informes locales. El objetivo será conocer todos los antecedentes sobre el estado de las poblaciones registradas, tanto en aspectos de distribución, come de abundancia para con la comparación de datos actuales y futuros, poder conocer el estado de las poblaciones y las alteraciones a que estén sujetas. Requerirá también la colaboración de otros Museos del extranjero, de Herpetólogos y Biólogos en general con registros de campo u observaciones que puedan ser útiles para este fin.

3. Estimular la formulación y realkación de proyectos que respondan a las necesidades de las medidas precedentes.

Actualmente el mayor esfuerzo de las investigaciones está dirigido a la realización de inventarios en un limitado número de localidades. Si bien es cierto, su aporte es importante, deben ampliarse a otras localidades. Por ejemplo es casi nulo el conocimiento que se tiene de las especies de grandes áreas de nuestro país (Fig. 2), y lo que es más grave aủn, sobre la mayoría de nuestras Unidades de Conservación, áreas a las que se debe dar prioridad. Igualmente se requieren investigaciones a largo plazo de poblaciones seleccionadas que reporten información en respuesta a las medidas sugeridas por el DAPTF. Se requiere reunir información básica necesaria sobre nuestras especies para la toma de decisiones de Conservación y Manejo.

\footnotetext{
4. Implementar nuevas medidas de Control de Manejo y Conservación de sus especies.

Se espera que como resultado de la ejecución de las medidas previas, se implementen nuevas medidas adecuadas de manejo y conservación de especies. A la fecha, todo aprovechamiento de especies ha sido netamente extractivo y sin ningún planearỉiento. Esta no constituye la única via de aprovechamiento, también se pueden aplicar programas de manejo en cautiverio o semi-cautiverio, o aún de poblaciones silvestres, tal como viene realizándose en Costa Rica, Brasil, Argentina, o
} 
Zimbawe. Para ello se requerirá la participación no únicamente de personal técnico o gubernamental, sino también la de exportadores, sin cuya colaboración las medidas no podrian ser efectivas.

\section{Implementar campañas de difusón para} corregir el posicionamiento mental de los anfibios y reptiles en el pública.

Los anfibios y reptiles son poco conocidos como especies de intenés o aprovechamiento; por el contrario son temidos por creencias culturales, más que por realidades prícticas (Estrada, 1994). Esto debe ser corregido brindando adecuada información sobre el valor estético de sus especies, el rol que cumplen en sus ecosistemas, sus increfbles aspectos de historia de vida, la facilidad y el valor que representan como indicadores de la alteración del medioambiente y por último, el valor comercial como recurso alimenticio, medicinal y comercial de sus especies conocidas y potencial de aquellas aún por descubrir. En otros cusos, las medidas de protección a determinadas especies apoyarín la de sus coosistemas, como en el caso de Iguana iguana, que habita ambientes ribereños en el nor-oocidente y amazonía peruana; o por su valor como indicadores del estado del medioambiente, como lo es en el caso de los anfibios, que poe su doble modo de vida, permite usarlos como indicadores de contuminantes en cuerpos de agua y aire (Jutterbock, 1990).

Larealización de las medidas precedentes, podráser lograda solocon la participación de entidades gubernamentales y nogubernamentales afines a la conservación, universidades, museos, investigadorese inclusive, público en general cuyas observaciones en el campo, puede constituir aporte significativo al conocimiento de nuestras especies.

Sólo de esta manera. podrá realizarse un adecuado aprovechamiento en investigación, conservacióa o manejo de nuestra berpetofauna como recurso, para nuestras necesidades presentes $y$ futuras.

\section{AGRADECIMIENTOS}

El autor desea expresar su mayor agradecimiento al Dr. Gerardo Lamas, Director del Museo de Historia Natural de la Universidad Nacional Mayor de San Marcos, por el apoyo y colaboración brindada; al Dr. William E. Duellman del Museo de Historia Natural de la Universidad de Kansas, quien colaboró con la mayor parte de la información presente; al Ing "Emilio Alvarezy Blg" Napolećn Castro, de la Dirección de Flora y Fauna Silvestre, por brindar facilidades para el conocimiento de nuestras exportaciones de fauna y regulaciones gubernamentales.

Por último, a la Dra. Nelly Carrillo de Espinoza, ex-Jefa del Departamento de Herpetologia del Museo de Historia Natural, bajo cuya turela y constante apoyo, se formb la presente generación de Herpetólogos del Museo.

\section{BIBLIOGRAFIA}

AICHINGER, M. 1987. Annual activity patterns of anurans in a seasonal neotropical environment. Oecologie 77-583-592.

AICHINGER, M. 1991. Faunal deficit of anurans in tropical farmland of Amazonian Peru. Alytes 9:23-32.

AICHINGER, M. 1994. A New Species of Poison-Dart Frog (Anura: Dendrobatidae) from the Serrania de Sira, Peru. Herpetologica, 47:1-5.

ANONIMO. 1976. Estudio Bioecologicode Ranas del Lago de Junin y Ensayo Experimental de su Crianza. Inf. Mecanog. Univ. Nac. Mayor de San Marcos y Minist. de Pesq. 47 págs.

ANONIMO. 1991. Declining amphibian populations - a global phenomenon? Findings and recommendations.Afytes 9.33-42.

ARANDA, C. y M. CHANDLER. 1989. Las Tontugas Marinas del Perú y su situación actual. Ból. Lima 1/ (62):77-86.

BLAUSTEIN, A. R. y D. B. WAKE. 1990a. Declining Amphibian Populations-A Global Pbenomenon? Ecol. Soc. of Amer. 71:127-18.

BLAUSTEIN, A. R. y D. B. WAKE. 1990b. Declining Amphibian Populations: A Global Phenomenon? Tree 5:203-204.

CADLE, J. E. 1989. A New Species of Coniophanes (Serpentes: Colubridae) from Northwestern Peru, Herpet. 45:411-424.

CADLE, J. E 1991. Reptiles y anfibios de los bosques montanos con referencia especial a los bosques de la Vertiente Oocidental en el noroeste del Perú.Bol. Mus. Hiss. Nat. Univ. Nac. May. San Marcos 3:4-5.

CADLE, J. E, y R. ALT7G. 1991. Two Lotic Tadpoles from the Andes of Southem Peru: Hyla armata and Bufo veraguensis, with Notes on the Call of Hyla amata (Amphibia: Anura: Hylidae and Bufonidae). Stud. Neotrop. Fauna Envir. 26:45-53.

CADLE, J. E. y P. CHUNA M. 1995. A New Lizard of the Gerus Macropholidus (Teidae) from a relictual humict forest of nonthwestern Peru, and notes on Macropholidus nuthveri Noble. Breviora Mus. Comp. Zool. 501:1-39.

CADLE, J. E. y R. W. MeDIARMID. 1990. Two New Species of Centrolenella (Anura: Centrolenidae) from Northwestem Peru. Proc. Biol. Soc. Wash. 103:746-768.

CANNATELLA, D. C. 1984. Two New Species of the LeptodactylidFrog genus Phrynopus, with comments on the 
phylogeny of the genus, Occ. Pap. Mus, Nat. Hist. Univ, Kansas /13:1-16.

CANNATELLA, D. C. y W. E. DUEL LMAN. 1982. Two New species of Centrolenella, with a brief review of the genus in Peru and Bolivia. Herpetologica 38:380-388.

CANNATELLA, D. C y W. E. DUELLMAN. 1984. Leptodactylid Frogs of the Physaldemas pustulosus Group. Copeia 1984,902-921.

CARRILLO DE ESPINOZA, N. 1968. Contribución al conocimiento de los Boideos Peruanos (Boidac, Ophidia, Reptilia). Reu Cien. 535-538:86-136.

CARRILLO DE ESPINOZA, N, 1970. Contribución al conocimiento de los Reptiles del Perú (Squamata, Crocodylią, Testudinata : Reptilia). PubL Mus. Hist. Nat. UNMSM (A)22:1-60

CARRIL.LO DE ESPINOZA, N, 1974. Sibynomorphus williamsii noy.sp. (Serpentes: Colubridac). Publ. Mus. Hist. nat UNMSM (A)24:1-16.

CARRILLO DE ESPINOZA. N. 1985. Contribución al conocimiento de las Serpientes Venenosas del Peni de las Familias Viperidae, Elapidae e Hjdrophiidae (Ophida: Reptilia), Pub, Mus. Hist. nat, UNMSM (A) 30,1-55.

CARRILLO DE ESPINOZA, N. y G. LAMAS. 1985. Un nuevo registro de tortuga terrestre para el Peri (Reptilia. Testudinata). Publ Mas. Hist nat. UNMSM (A)3l:1-7,

CARRILLO DE ESPINOZA, N. 1987. Hallazgo de Eretmochelys imbricata bissa (Ruppeb) en la Costa Norte del Peru (Testudinata: Chelonidae). Biofa 94:40-45

CARRILLO DE ESPINOZA, N. 1990. Nombres populares de los Reptiles del Perí. Bol. Lima 70:23-28.

CARRILLOO DE ESPINOZA, N. D. ROTHENSTEIN, A W. SALAS, F. VIDEL.A and Y. L. WERNER. 1990. Radiation and Convergence Among Desert Geckos : Phyliodactylus species resembling boh Pryodactylis and Stenodactylus. Amphibia-Reptilia |l:1-13.

CENTRO DE DATOS PARA LA CONSERVACION . UNA. 1990. Especies amenazadas de la Fauma Silvestre Peruana- Clasificación Oficial. 10 págs.

COCROFT, R. B. y K. HAMBLER. 1989, Observations on a commensal relationship of the microhylid frog Chiarmocleis ventrimaculata and the burrowing theraphosid spider Xenesthis immanis in southern Peru. Biotrop. 2/:2-8.

CONSERVATION INTERNATIONAL, 1994. The Tambopata-Candano Reserved Zone of Southeastem Peni: A Biological Assessment, Rapid Assessment Program. RAP Working Papers 6, 184 págs.
CUTTI O., F. y H. V. ASCENCIOS. 1990 . Observaciones sobre nacimientos en cautiverio de serpientes venenosas peruanas. Bol. Lima 71: 33-40.

De SA, R. O. y L. TRUEB. 1991. Osteology, skeletal Development and Chondrocranial Structure of Hampropinne boliviana (Anura Microhylidac). Jour. Morph. 209:311-330.

DIXON, J, R. 1973. A systematic review of the teiid lizards, genus Bachia, with remarks on Heterodactylus and Anotosaura. Univ, Kansas Mus. Nat. Hist. Misc. PubL. $57: 1-47$,

DLXON, J. R. 1974, Systematic review of the microteiid genus Iphisa. Herpetol. 30:133-139.

DIXON, J. R. 1979. Origin and Distribution of Reptiles in Lowland Tropical Rainforests of South America, In W. E. Dueliman (ed.), The South American Herpetofauna: its origin, evolution and dispersal, pp. 217-240. Monog. Mus. Nat, Hist, Univi Kansas 7:1-485.

DIXON, J. R. y R. B. HUEY. 1970. Systematics of the lizards of gekkonid genus Phyllodactylus of mainland South America. Los Angeles Country Mus. Contrib. Sci. 192:1-78

DIXON, J. R. y P. SOINI. 1975. The reptiles of the upper Amazon Basin, Iquitos region, Peru. L Lizards and amphisbacnians. Contrib. Biol, Geol. Mitwaukee Publ Mus, 4:1-58.

DIXON, J. R. y P. SOINI. 1977. The reptiles of the upper Amazon Basin, Iquitos region, Peru. II. Crodoxdilians. turtles and snakes. Contrib. Biol. Geol. Milwaukee Publ. Mus, 12:1-91.

DIXON, J. R. y J. W. WRIGHT. 1975. A review of the lizards of the genusTropidunes in Peru. Nat. Hist. Mus. Las Angeles Cty. Conrrib. Sci. 192:1-78.

DUELLMAN, W. E. 1973. Descriptions of New Lizards from the Upper Amazon Basin. Herpetologica 29;228231.

DUEL.LMAN, W. E. 1976. Centrolenid frogs from Peru. Occ. Pap. Mus. Nat. Hist. Unin. Kansas 52:1-11.

DUELLMAN, W. E. 1978a. Two new species of Eleutherodactylus (Anura: Leptodactylidae) from the Peruvian Andes. Trane Kansas Acad Sct 81,65-71.

DUELLMAN, W. E. 1978b. New species of leptodactylid frogs of the genus Eleutherodactylius from the Cosnilipata Valley, Peru. Proc Biol. Soc, Wash. 91:418-430.

DUELLMAN, W. E. 1979. The Herpetofauna of the Andes: patterns of distribution, origin, differentiation and 
present communities. In W. E. Duellman (ed.), The South American Herpetofauna: is origin, evolution and dispersal, pp. 371-459. Monog. Mus. Nat, Hist. Univ, Kansas 7:1-485.

DUELLMAN, W. E. 1982. A New Species of Small Yellow Hyla from Peru (Anura: Hylidae). AmphibiaReptilia 3:153-160.

DUELLMAN, W.E. 1987a, Lizards in an Amazonian rain forest community: resource utilization and abundance.Nat. Geog. Res. 3:489-500.

DUEllmaN, W. E. 1987b. Two New Species of Marsupial Frogs (Anura: Hylidae) from Peru. Copeia 4:903-909.

DUELlmaN, W. E. $1990 \mathrm{a}$. A New Species of Eleutherodactylus from the Andes of Northern Peru (Anura: Leptodactylidae), Jour. Herp. 24:348-350.

DUELlMaN, W. E. 1990b. A New Species of Leptodactylid Frog, Genus Ischnocnema, from Peru. Occ. Pap. Mus. Nat. Hist. Univ, Kansas 138:1-7.

DUELLMAN, W. E. 1991a. A New Species of Eleutherodactylus (Anura: Leptodactylidae) from the Condillera Oocidental of Peru. Herpetol. 47:6-9.

DUELLMAN, W. E. 1991b. A New Species of Leptodactylid Frog, Genas Phyllonastes, from Peru. Herpetol 47:9-13.

DUELLMAN, W. E. 1992a. A New species of the Eleutherodactylus conspicillatus Group (Anura: Leptodactylidac) from Northeastern Peru. Rev. Esp. Herp. 6:23-29.

DUELLMAN, W. E. 1992b. Eleutherodactylus bearsei New Species (Anura: Leptodactylidac) from Northeastern Peru. Occ, Pap, Mus. Nat. Hist Kansas 150:1-7

DUELLMAN, W. E, J.E. CADLEy D. CANNATELLA. 1988. A new species of terrestrial Phyllomedusa (Anura: Hylidae) from southern Peru. Herpetologica 44:91-95.

DUELLMAN, W. E, y R. De SA. 1988. A new genus and species of South American Hylid Frog with a highly modified tadpole. Trop. Zool. 117-136.

DUELLMAN, W.E.y T. H. FRITTS, 1972. A Taxonomic Review of the Southern Andean Marsupial Frogs (Hylidae: Gastrorheca). Occ. Pap. Mass. Nat. Hist, Univ, Kansas 9:1-37.

DUELLMAN, W. E. y V. MORALES. 1990. Variation, distribution and life history of Edalorhina perezi (Amphibia, Anura, Leptodactylidae). Stud. Neotrop. Fauna Envir. 25:19-30.
DUELLMAN, W. E y O. OCHOA. 1991. A New Species of Bufo (Anura Bufonidac) from the Andes of Southem Peru. Copeia I99I(:137-14L.

DUEL IMAN, W. E. y A. W. SALAS. 1991. Annotated Checklist of the Amphibians and Reptiles of Cuzco Amazonico, Peru. Occ. Pap. Mus. Nat. Hist Univ. Kansas 143:1-13.

DUELLMAN, W. E. y R. SCHULTE, 1992. Description of a New Species of Bufo from Northern Peru with Comments on Phenetic Groups of South American Toads (Anura: Bufonidae), Copeia 1992:162-172.

DUELLMAN, W. E y R. SCHULTE. 1993. New Species of Centrolenid Frogs from Northem Peru, Occ, Pap. Mus. Nat. Hist. Univ. Kansas 155:1-313.

DUELLMAN, W. E. y C. A. TOFT. 1979. Anurans from the Serrania de Sira, Peru: taxonomy and biogeography. Herpetologica 35-60-70.

DUELLMAN, W. E. y L. TRUEB. 1988. Cryptic species of Hylid Marsugial Frogs in Peru. Jour. Herpet. 22:159-179.

DUELLMAN, W. E. y L. TRUEB. 1989. Two new treefrogs of the Hyla parviceps group from the Amazon Basin in southern Peru. Herpetologica 45:1-10.

DUELLMAN, W. E y A. VELOSO. 1977. Phylogeny of Pleurodema (Anura : Leptodactylidac): A Biogeographic Model. Occ. Pap, Mus. Nat. Hist. Univ. Kansas 64:1-46.

DUELIMAN, W. E y J. J. WIENS. 1992. The Status of the Hylid Frog Genus Olalygon and the Recognition of Scinax Wagler, 1830. Oec. Pap. Mus. Nat. Hist. Kansas 151:1-23.

DUELLIMAN, W, E, y J. J. WIENS. 1993. Hylid Frogs of the Genus Scinax Wagler, 1830, in Amazonian Ecuador and Peru. Occ. Pap. Mas. Nat. Hist. Kansas 153:1-57.

DUELLMAN, W. E, y E. R. WILD. 1993. Anuran Amphibians from the Cordillera de Huancabamba, Northem Peru: Systematics, Ecology, and Biogeography. Occ. Pap. Mus. Nat. Hist. Kansas 157:1-53.

ESTRADA C. M. 1994. Mitos y Leyendas Amazónicas. Centro de Estudios Teológicos de la Amazonia, Iquitos, Perù. 23 págs,

FACHIN, A. 1991. Las tortugas de la Reserva PacayaSamiria, un recurso amenazado. Kanatari $B(351): 3$.

FACHIN, A. 1992. Desove y Uso de Playas para Nidificación de Taricaya (Podocnemis unifilis) en el Rio Samiria, Loreto-Peru. Bol. Lima 79:65-75. 
FRITTS, T. H. 1974, A multivariate evolutionary analysis of the Andean iguanid lizards of the genus Srenocercus. San Diego Soc. Nat. Hist. Mem. (7):1-89.

FROST, D. 1986, Amphibian Species of the World. Lawrence, Kansas: Assoc. Syst. Coll.

GRAY, P. y D. C. CANNATELLA. 1985. A New Species of Atelopis from the Andes of northern Peru. Copeia. 1985:910-917.

GRUSON, L. 1989. A Plan to Save Iguana, and the Rain Forests in the Bargain. The New York Times. Aug. 22, 1989. Pág. 1-2.

GUEVARA, M. E. y V.R. MORALES. 1991. Nota sobre la composición algal de la dieta en larvas de Batrachophrynus Peters, 1873 (Anura: Leptodactylidac) del Perú. Publ. Mus. Hisz. nar. UNMSM (A) 47:6-7.

HEDGES, S. B. 1990. A New Species of Phrynopus (Anura: Leptodacylidae) from Perí, Copeia (1):108-112

HEDGES, S. B. y A. SCHLUTER. 1992. Eleutherodactylus eurydactylus, a New Species of Frog from Central Amazonian Pent (Anura: Leptodactylidae) Copeia (4): $1002-1006$

HENLE, K. 1991. Ololygan pedromedinate sp, nov, ein neuer knickzehenlaubfrosch (Hylidac) aus Peru. Salamandra 27:76-82.

HERTWIG, L, y U. SINSCH. 1995, Comparative Toe Pad Morphology in Marsupial Frogs (Genus Gastrotheca): Arboreal versus Ground-Dwelling Species. Copeia (1):38-47.

HERRON, Jon C 1985. Population Status, Spatial Relations, Growth, and Injuries in Black and Spectacled Caimans in Cocha Castu. Tesis presentada para la obtención del Grado de Bachiller en Artes, Departamento de Biología, Universidad de Princeton.

HEYER, W. R. 1994, Variation within the Leptodactylas podicipinas-wagner Complex of Frogs (Amphibia: Leptodactylidae), Smith. Contrib. Zoot. 546; 1-124.

HEYER, R. W, y V. R. MORAI FS. The advertisement call of the leptodactylid frog Leptodacrylus griseigularis. Amphibia-Repsilia 16:91-92.

HILLIS, D. M. y R. De SA. 1988. Phylogeny and Taxonomy of the Rana palmipes group (Salientia: Ranidae), Herpet. Monog, 2-1-26.

HUEY, R. B. 1974. Winter Thermal Ecology of the igunnid lizard Tropidunus peruvianus. Copeia 1974:149-155.

HUEY, R. B. 1979. Parapatry and niche complementarity of Peruvian desert geckos (Phyllodatylus): the ambiguous role of competition. Oecologie (Berl) 38:249-259.
HUTTON, J.M. and G.F.T. CHILD. 1989. Crocadile Management in Zimbabwe. In: Crocodiles. IUCN Publ. N.S., Gland, ISBN 2-8023-209-X. Pgs. 62-79.

ICOCHEA, J. 1992. Herpetofauna del Santuario Nacical Pampas del Heath, Madre de Dios, Peru: Diversidad y Conservación. Memoria X Conabiol, 02-07 Agosto 1992. Lima-Peru. pp. 351-354.

IUCN/SSC Task Force on Declining Amphibians 1992. Froglog. $\mathrm{N}^{2} 1.4$ págs.

JUTTERBOCK, J.E. 1990. SSAR Annual Meeting Report New Orleans 1990. Herp. Review $21: 70-76$.

LESCURE, J. y J. P. GASC 1986. Partage de I'Espace forestier par les amphibiens et les reptiles en Amazonie du Nord-Ouest. Caldasia 15:707-723.

LYNCH, J. D. 1975. A revision of the Andean leptodactylid frog genus Phrynopus. Occ. Pap. Mus. Nat. Hiss. Univ. Kansas 35:1-51.

LYNCH, J. D, y J. LESCURE. 1980. A collection of eleutherodactyline frogs from northeastern Amazonian Peru with the descriptions of two new species (Amphibia. Salientia, Leptodactylidae). Bull. Mus. Natl. Hist. Nat. (4) $2 A: 303-316$.

McNEEIY,J., K. MILLER, W. REID, R. MITTERMEIER y T. WERNER, 1990, Conserving the World's Biological Diversity. IUCN, Gland, Switzerland, WRI, Cl, WWF-US, World Bamk, Washing, D.C., 193 págs.

MITTERMEIER, R.A., A. G.J.RHODIN.,F.MEDEM,P. SOINI, M. S. HOOGMOED y N. CARRILLO, 1978. Distributicn of the South American Chelid Turtle Phrynops gibbus, with observationns on habitat and reproduction. Herpetologicu 34:94-100.

MONTANUCCI, R. R. 1973, Systematics and evolution of the Andean lizand genus Pholidabolus (Sauria: Teidae). Univ. Kansas Mus. Nat Hist. Misc Publ. 59:1-52.

MORALES, V, R. 1988. Estudio de la Herpetofauna Anura en dos localidades del Departamento de Ancash. Tes. Bach. CC. BB. U.R.P., 53 pp. Lima.

MORALES, V. R. 1988. Una Nueva Especie de Telmatobius (Anura, Leptodactylidae) de Ancash, Peru. Rev. Bras. Zool 5:603-608.

MORALES, V. R. 1991. Anfibios y Reptiles en las Pampas del Heath. El Peruano, Rev. I-II. Marzo 25.

MORALES, V. R 1992. Dos Especies Nuevas de Dendrobates (Anura: Dendrobatidae) para Peri. Joumal of Science 28:191-199, 
MYERS, C. W, y J. W. DALY, 1979. A Name for the Poison Frog of Cordillera Azul, Eastern Peru, With Notes on Its Biology and Skin Toxins (Dendrobatidae). Am. Mus. Novitates 2674:1-24.

PEFAUR, J.E. Y W. E. DUELLMAN, 1977, Community structure in High Andean herpetofaunas, Herpetol Rev. 8: Suppl. 6-7.

PEFAUR, J. E. y E. LOPEZ-TEJEDA 1983. Ecological notes on the lizard Tropidurus perivianus in southern Peru. Joumal of Arid Environments 6:155-160.

PEFAUR, J. E, A, NUÑEL., E. LOPEZ, J. DAVILA. 1978a. Distribución y Clasificación de los Anfibios del Departamento de Arequipa. Bull. Inst. Fr. E2. And. 7:1 19-127.

PEFAUR, J, E., A. NUÑEL, E. LOPEZ, J. DAVILA. 1978b. Distribucion y Clasificación de los Reptiles del Departamento de Arequipa. Bull, Inst. Fr. Er. And. 7:129-139.

PETERS, J. A. y R. DONOSO-BARROS. 1970. Cataloguc of the Neotropical Squamata: Part II. Lizards and amphisbaenians. Bull. U.S. Natl. Mus. 297:1-293.

PETERS, J. A. y B. OREJAS-MIRANDA. 1970. Catalogue of the Neotropical Squamata: Part I. Snakes. Bull, U.S. Natl. Mus. 297:1-347.

PHILLIPS, K. 1990. Where have all the frogs and toads gone? Bioscience 40:422-424.

RODRIGUEZ, L, O. 1992, Structure et organisation du peuplement d'Anoures de Cocha Cashu, Pare National Manu, Amazonie Péruvienne, Rev. Ecol. 47:151-197.

RODRIGUEZ, L. O, 1994. A new species of the Eleutherodactylis conspicillatis group (Leptodactylidae) from Peru, with comments on its call. Alyres 12:49-63.

RODRIGUEZ, L. B. y J. E. CADLE, 1990. A preliminary of the herpetofauna of Cocha Cashu, Manu National Park, Peri. Pp. 410-425 in A. H. Gentry (ed.), Four Neotropical Rainforests. New Haven: Yale Univ. Press.

RODRIGUEZ, L. O. y C. W. MYERS. 1993. A New Poison Frog from Manu National Park, Sooutheastern Peru (Dendrobatidae, Epipedobates). Am. Mus. Novitates 3068:1-15.

RODRIGUEZ, L. O, J. H. CORDOVA Y J. ICOCHEA. 1993. Lista Preliminar de los Anfibios del Peru. Pub. Mus. Hist. nat. UNMSM (A) 45:1-22.

ROJAS, M. C. 1991. Liberan 1000 iguanas en Turrubares. La Nación. Enero 29. Pagg. 2A.

SALAS, A W. 1986. Proyecto Explotación y Fomento de la Rana Batrachophrynus macrostomus. Inf. Final a Corde-Junin y Dir. Reg. MIPE. 10 págs.
SAL.AS, A. W. 1990a. Evaluación de las Exportaciones de Anfibios y Reptiles, Peru. Inf. Prelim, a la D. G. F.F. (M. A.). 8 págs.

SALAS, A, W, 1990b. Observaciones Preliminares sobre la Ecologia y Conducta Reproductiva del Telmatobisis carrillae Morales 1988 (Anura: Leptodactylidae). Tes. Bach., CC. BB, U, R. P, 57 págs. Lima.

SALAS, A W. 1991a. Experimentación de Nuevas Metodologias Standarizadas para la Determinación de la Biodiversidad y Ahundancia de Herpetofauna, E. B. Cocha Cashu, Parque Nacional del Manu. Inf. Prelim. aja D. G. F. F. (M. A.), 18 pags.

SAL_AS, A. W. 1991b. Estimación de Abundancia de la Herpetofauna del Nor-Occidente Peruano. Inf. Prelim. a la D. G.F.F. (M. A.). 10 págs.

SALAS, A. W. (En preparación), Geckos del Perú: Lista de Especies y su Distribución.

SALAS, A. W, y U, SINSCH. (en prensa), Two New Telmatobius species (Leptodactylidac: Telmatobiinae) of Ancash, Perí. Alytes.

SCHLUTER, A. 1979. Bio-akustische Untersuchungen an Hyliden in einem begrenzten Gebiet des tropischen Regenwaldes von Peru (Amphibia: Salientia: Microbylidae). Salamandra /5:211-236.

SCHLUTER, A. 1980. Bio-akustische Untersuchungen an Microhylidae in einem begrenzten Gebiet des tropischen Regenwaldes von Peru (Amphibia: Salientia: Microhylidae), Salamandra 16:114-131.

SCHLUTER, A. 1983, Okologische Untersuchungen an einem Stiligewăsser im tropischen Regenwald von Peru unter besorender Berilicksichtigung der Amphibien, Duct. Diss. Univ. Hamburgo. 300 pag.

SCHLUTER, A. 1987, Dendrophrnicus minutus, Reproduction. Herp. Review 18:33.

SCHLUTER, A. 1990. Reproduction and tadpole of Edalorhina perezi (Amphibia, Leptodactylidac). Stud. Neatrop. Fauna Emvinon., 25:49-56.

SCHLUTER, A y A. W. SALAS, 1990. Comparative Study on Reproduction, Tadpoles and ecology of Three Sympatric Microhylid Species from Peru (Anura:Microhylidae). Stuttgarter Beitrage zur Naturkunde, Ser, A 458:1-17.

SCHULTE, R, 1988. Observaciones sobre la boa verde, Corallus caninus, en el Departamento San Martín- Perú. BoL Lima 55:21-26, 
SINSCH, U. 1985, Die Reproduktionsbiologie eines bachbewohnenden Frosches, Telmatobius jelskii, in einem Andenhochtal Zentralperus, Veri. Deut. Zool. Ges. Wien 1985:265.

SINSCH, U, 1986, Anfibios de la Sierra Central del Peru - Una clave de identificación para adultos y larvas, Bol, de Lima 45:399-407,

SINSCH, U. 1988a, El sapo andino Bufo spinulosus: analisis preliminar de su orientación hacia sus lugares de reproducción. Bol de Lima 57:83-91.

SINSCH, U. 1988b. EinfluB von Temperatur und Ernahrung auf die diurnale Rufaktivitait des Beutelfrosches, Gastrotheca marsupiata. Verh. Deut. Zool. Ges. Bielefeld 81:263-264.

SINSCH, U, 1989. Behavioural Thermoregulation of the Andean Toad (Bufo spinulosus) at high altitudes. Oecologia 80:32-38.

SINSCH, U. 1990. Froschlurche (Anura) der zentralperuanischen Andea: Artdiagnose, Taxonomic, Habitate, Verhaltesibkologic. Salamandra 26:177-214.

SINSCH, U, 1991. Análisis radio-telemétrico de la regulación térmica del sapo andino, Bufo spinulasus. Boletín de Lima 73:65-73,

SINSCH, U. y G. JOERMANN. 1989, Vocalization and mating bebaviour of the marsupial frog. Gastrotheco marsupiata (Dumeril and Bibron), 1841) (Amphibia: Hylidae). Copeia 1989:750-755.

SINSCH, U. A. W. SALAS y V, CANALES. 1995. Reassessment of central Peruvian Telmatobiinae (genera Batrachophrymus and Telmatobies). L. Morphometry and classification. Alyfes $13(1): 14-44$.

SOINI, P. 1994. Ecologia Reproductiva (Podocnenis tanifilis) en el Rio Pacaya, Peni. Fotia Amazónica 6:105-124

SOINI, P. M. USHIÑAHUA, M. TRIGOSO Y L. MOYA. 1989. Los quelonios acuáticos de la Amazonia Peruana (1). Kanatari 6(250):24
TITUS, T.A., D, M. HIL.L.IS y W, E. DUELLMAN. 1989. Color polymorphism in neotropical treefrogs: an allozymic investigation of the taxonomic status of $\mathrm{Hyla}$ favasa Cope, Herpetologica 45:17-23.

TOFT, C. A. 1980. Feeding Ecology of Thirteen Syntopic Species of Anurans in a Seasonal Tropical Environment. Oecologia (Berl.) 45:131-141.

TOFT, C.A. $y$ W. E. DUELLMAN. 1979. Anurans of the lower Rio Llullapichis, Amazonian Perú: A Preliminary analysis of community structure. Herper. 35:71-77.

TRUEB, L. A. 1974, Systematics Relationships of Neotropical Horned Frogs, Genus Hemiphractus (Anura : Hylidac), Occ. Pap. Mus. Nat. Hist. Univ, Kansas 29:1-60.

VANZOLINI, P. E. 1986. Addenda and corrigenda to the catalogue of neotropical Squamata. Smithsonian Herpetol. Infor, Serv, 70:1-25.

VASQUEZ, R. P. 1983. Análisis de la Simuación Acual de los Caimanes y del Cocodrilo de Tumbes en el Peru. Rev. Forest, del Peri 11:171-187.

VIAL, J. L y L. SAYLOR. 1993. The Status of Amphibian Populations, A Compilation and Analysis. IUCN/SSC Declining Amphibian Populations Task Force. Working Document $N^{*} 1.98$ págs,

WAKE, D. B. 1990. Declining Amphibian Populations. Science 253:860.

WIENS, J, 1993. Systematics of the leptodactylid frog genus Telmatabius in the Andes of northern Peru. Occ. Pap. Mus. nat. Hist, Univ, Kansas, 162:1-76.

WORLD RESOURCES INSTTTUTE, UNEP y UNDP. 1990. World Resources 1990-91. Oxford Univ, Press

ZIMMERMANN, H. y E. ZIMMERMANN. 1988. EthoTaxonomie und zoogeographische Artengruppenbildung bei Pfeilgifufrüschen (Anura: Dendrobatidae), Salamandra 24:125-160. 